\title{
Capturing the $\mathrm{H}_{2}-$ Metal Interaction in the M-MOF-74 Series Using Classical Polarization
}
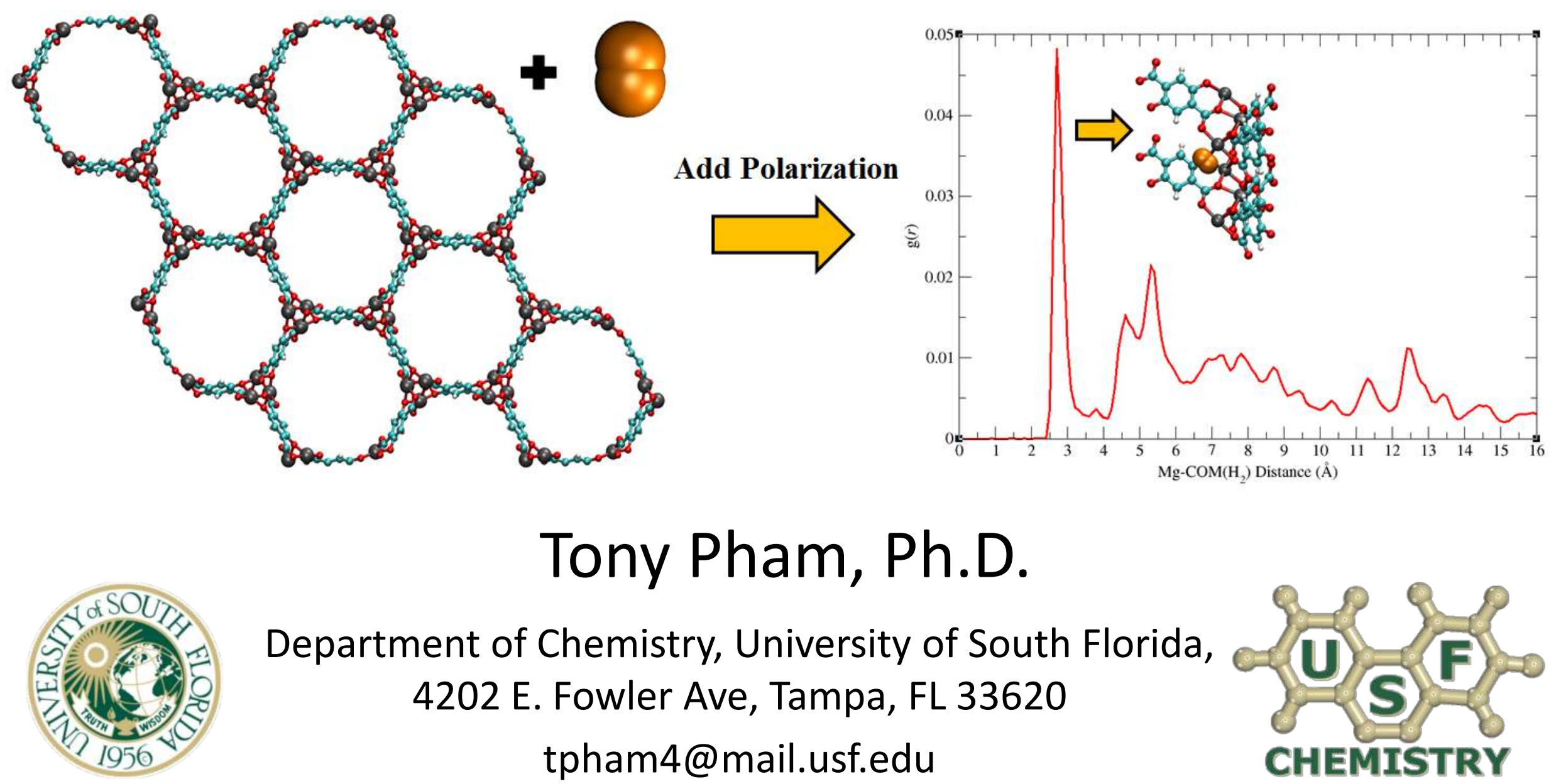

\section{Tony Pham, Ph.D.}

Department of Chemistry, University of South Florida,

4202 E. Fowler Ave, Tampa, FL 33620 tpham4@mail.usf.edu

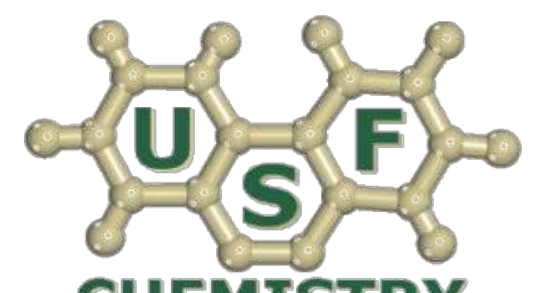
CHEMISTRY 


\section{Hydrogen Storage}

- Advantages:

- Abundant in nature

- Releases only $\mathrm{H}_{2} \mathrm{O}$ as a byproduct

- Releases $120 \mathrm{~kJ} \mathrm{~g}^{-1}$ of energy

- Challenges:

- Interacts weakly with environment

- Liquefies at $20 \mathrm{~K} / 1 \mathrm{~atm}$

- Requires large tank for storage. 


\section{Metal-Organic Frameworks}

- Metal-organic frameworks (MOFs) are a class of porous crystalline materials that consists of metal ions coordinated to organic ligands.

- MOFs have been shown to be promising candidates for $\mathrm{H}_{2}$ storage.

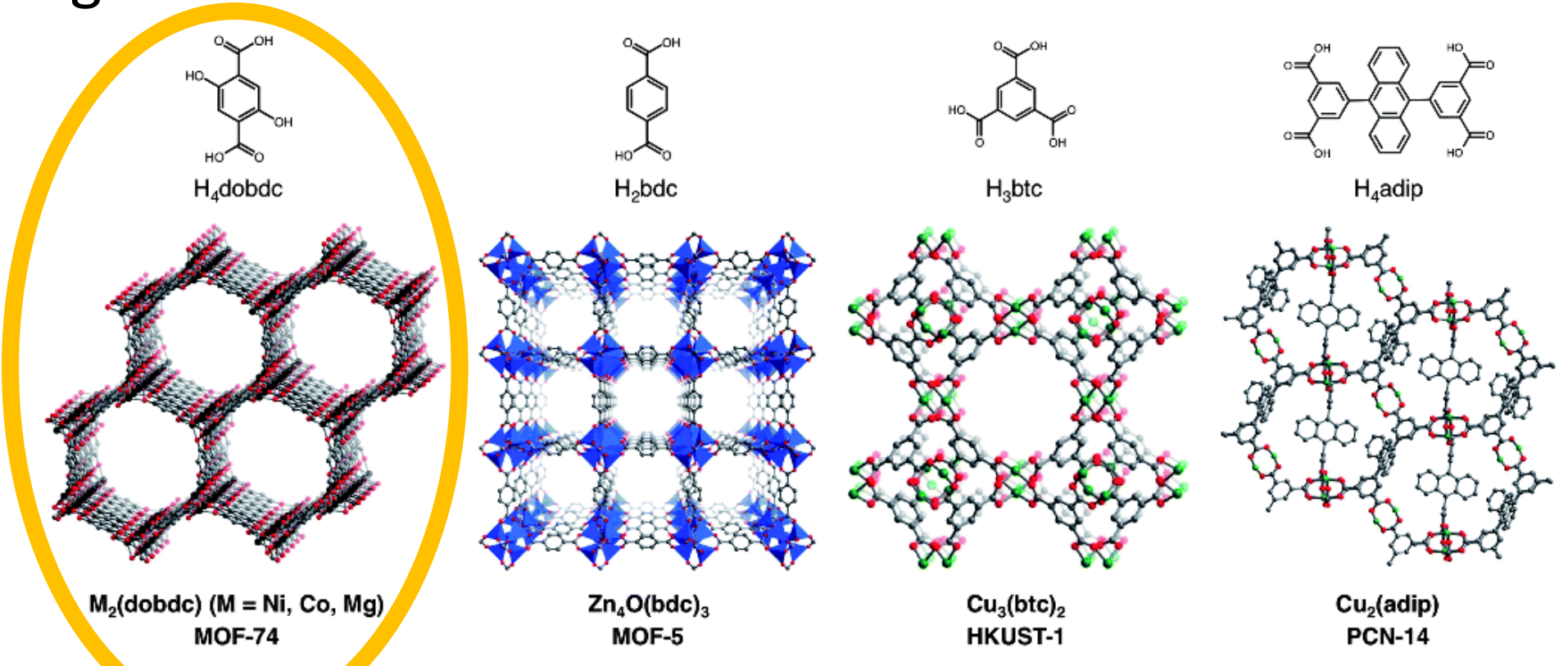


1.) Caskey, S. R.; et al. J. Am. Chem. Soc. 2008, 130, 10870-10871.

2.) Zhou, W.; et al. J. Am. Chem. Soc. 2008, 130, 15268-15269.

\section{M-MOF-74}

3.) Dietzel, P. D. C.; et al. Chem. Commun. 2010, 46, 4962-4964.

4.) Rosnes, M. H.; et al. J. Mater. Chem. A 2015, 3, 4827-4839.
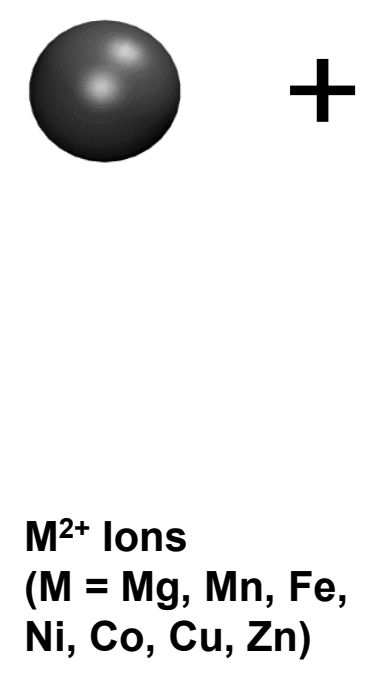

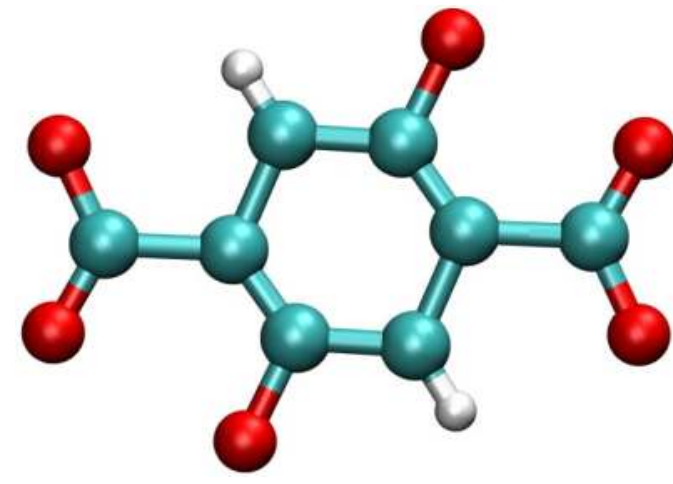

2,5-dioxido-1,4benzenedicarboxylate (dobdc)

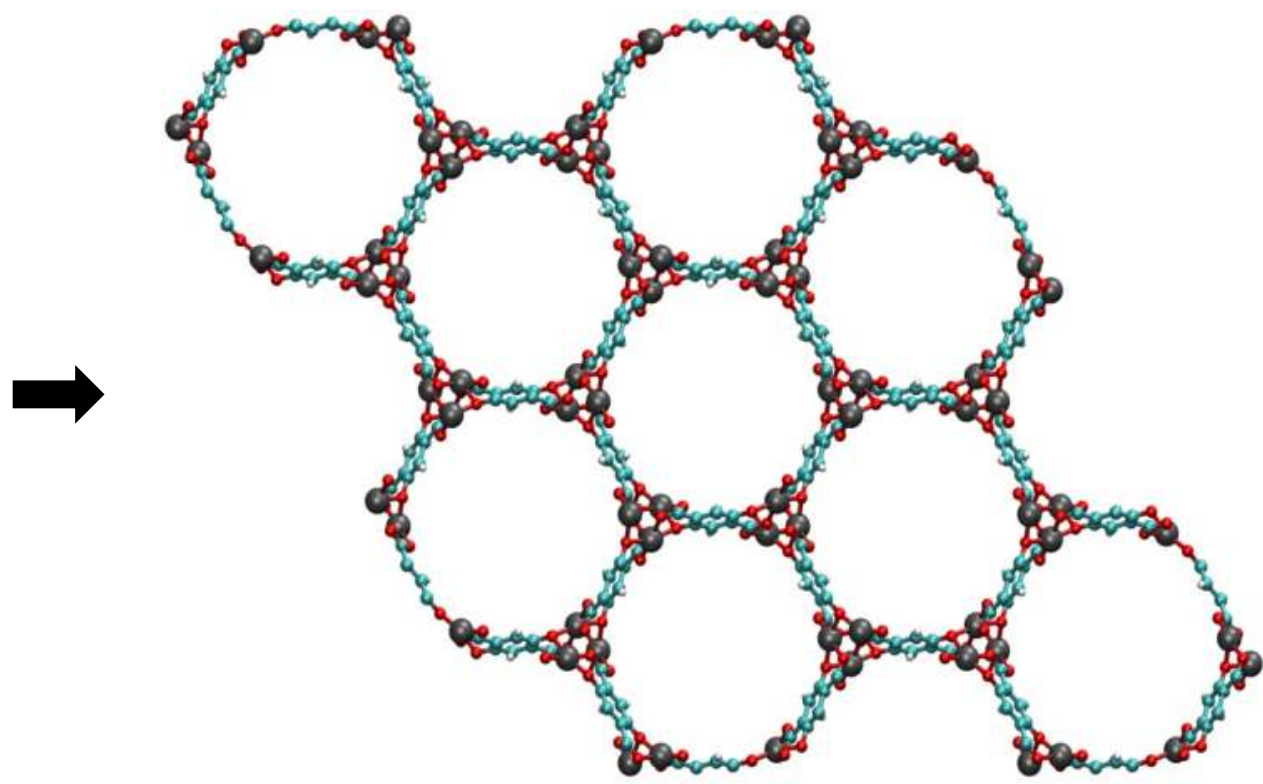

M-MOF-74 


\section{Experimental $\mathrm{H}_{2}$ Adsorption Results in M-MOF-74}

Mg-MOF=74 $=$ red Ni-MOF-74 = blue Co-MOF-74 $=$ violet
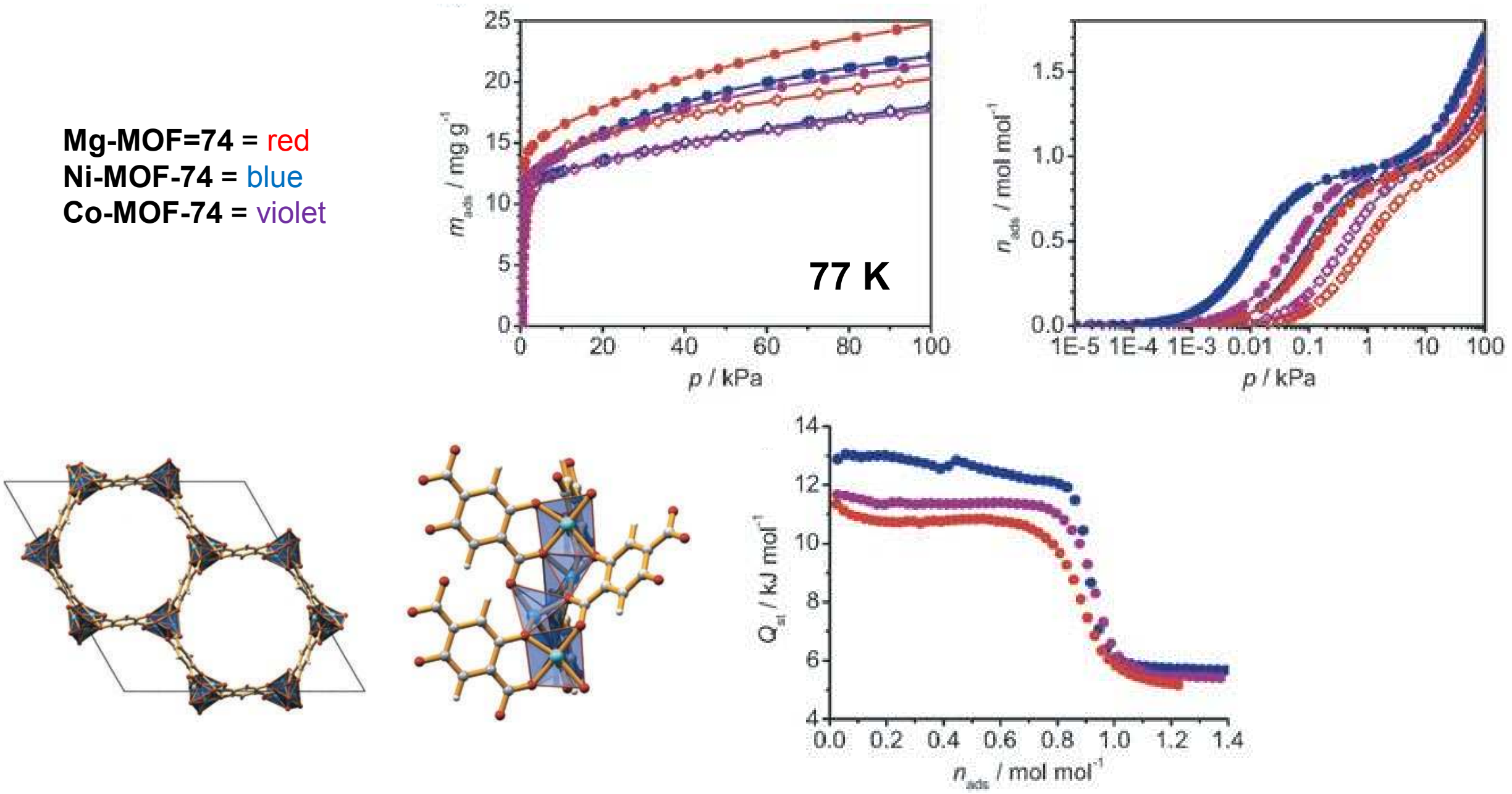

Dietzel, P. D. C.; Georgiev, P. A.; Eckert, J.; Blom, R.; Strässle, T.; Unruh, T. Chem. Commun. 2010, 46, 4962-4964. 


\section{Simulation Parameters}

- Repulsion/Dispersion

$$
U_{r d}=4 \varepsilon_{i j}\left[\left(\frac{\sigma_{i j}}{r_{i j}}\right)^{12}-\left(\frac{\sigma_{i j}}{r_{i j}}\right)^{6}\right]
$$

- Lennard-Jones (12-6 potential) parameters were taken from the Universal Force Field (UFF).

- Electrostatics

- Partial charges were determined through fitting to the electrostatic potential surface of gas phase fragments.

- Polarization

- Atomic point polarizabilities were taken from van Duijnen, P. T.; Swart, M. J. Phys. Chem. A 1998, 102, 2399-2407.

$$
U=U_{r d}+U_{\text {elec }}+U_{\text {pol }}
$$




\section{Gas Phase Fragments}
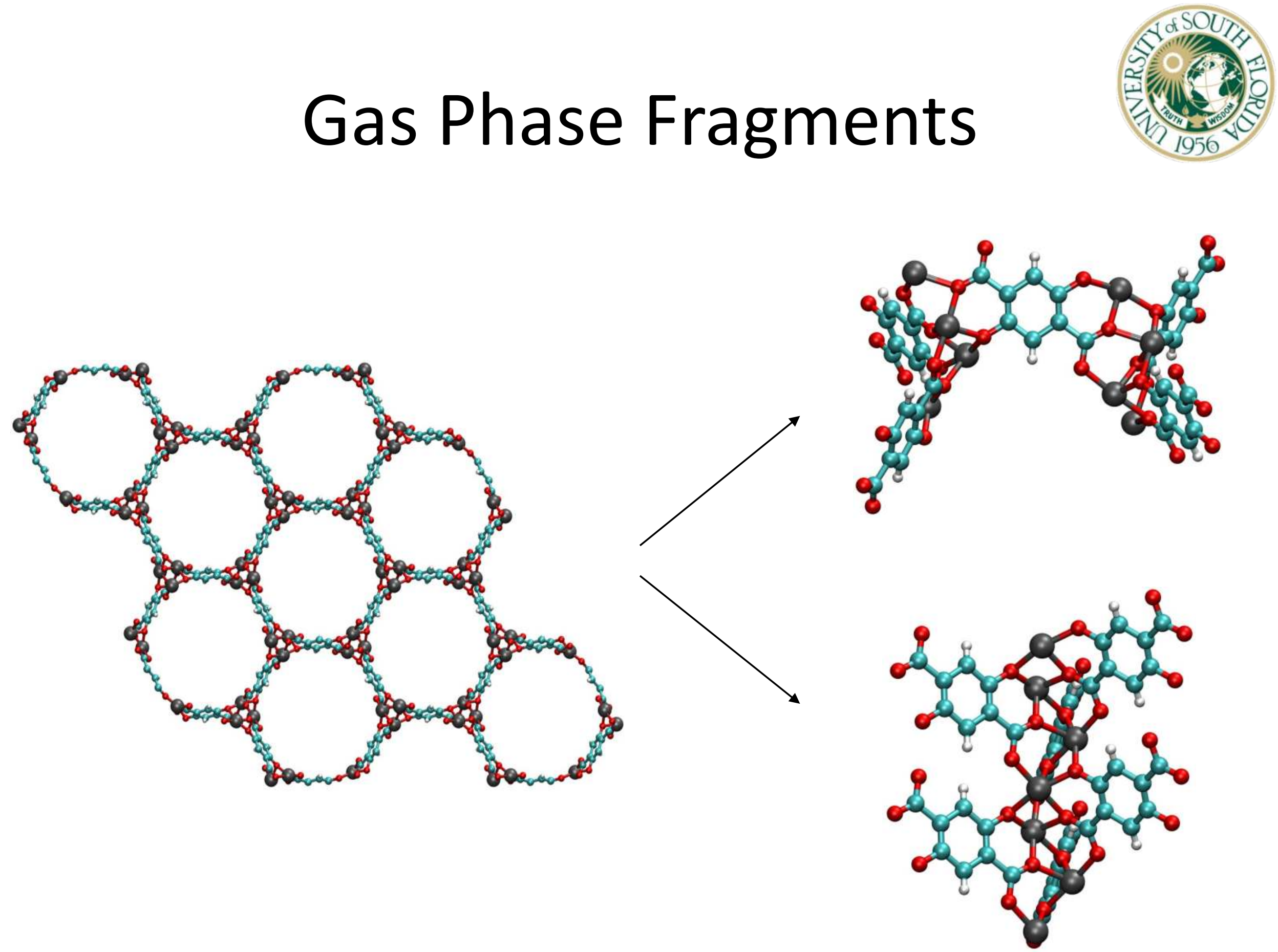


\section{Chemically Distinct Atoms}

(5)

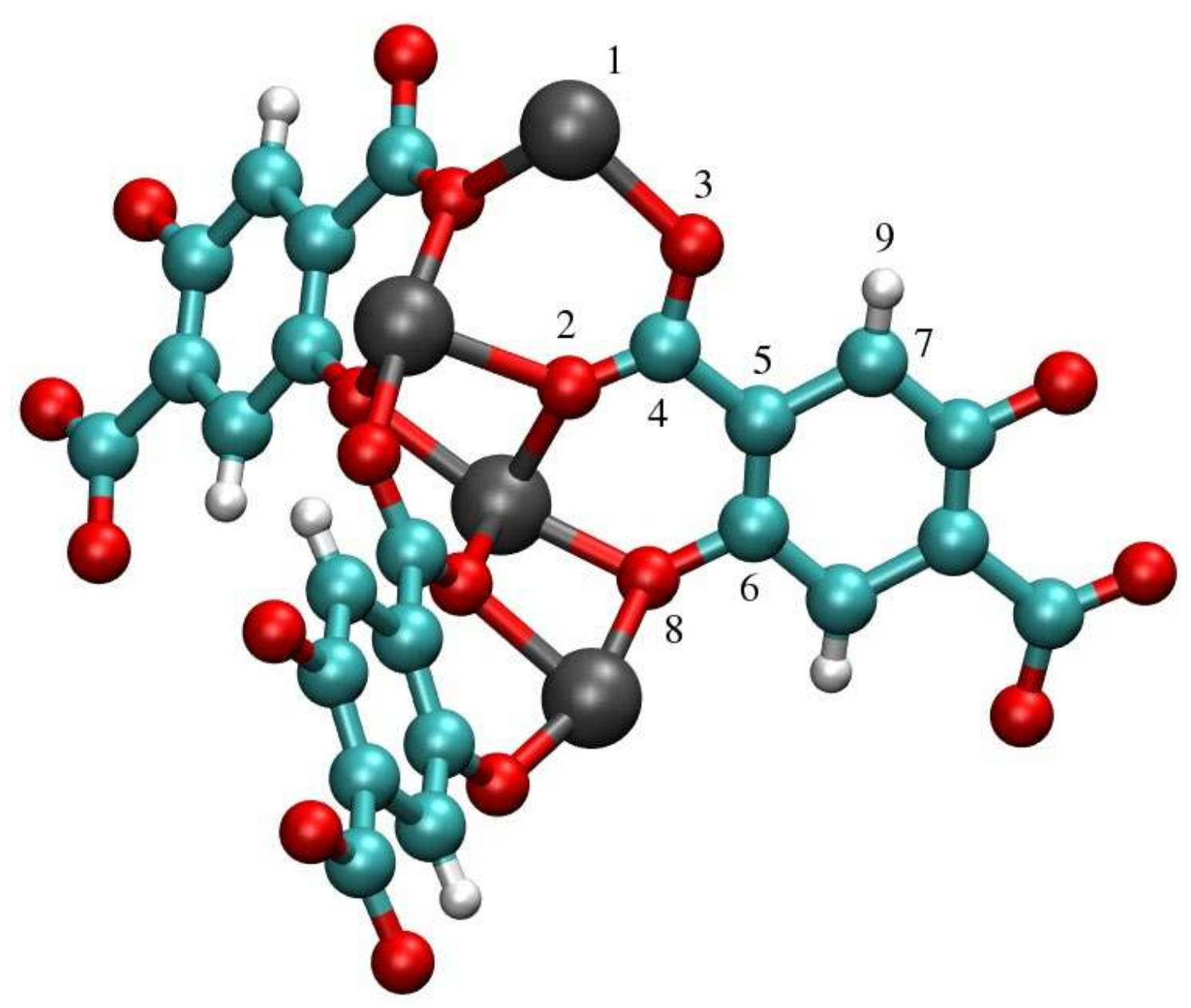


1.) Applequist, J.; Carl, J.

R.; Fung, K.-K. J. Am.

Chem. Soc. 1972, 94, 2952-2960.

2.) Thole, B. Chem.

Polarization

Phys. 1981, 59, 341-350.

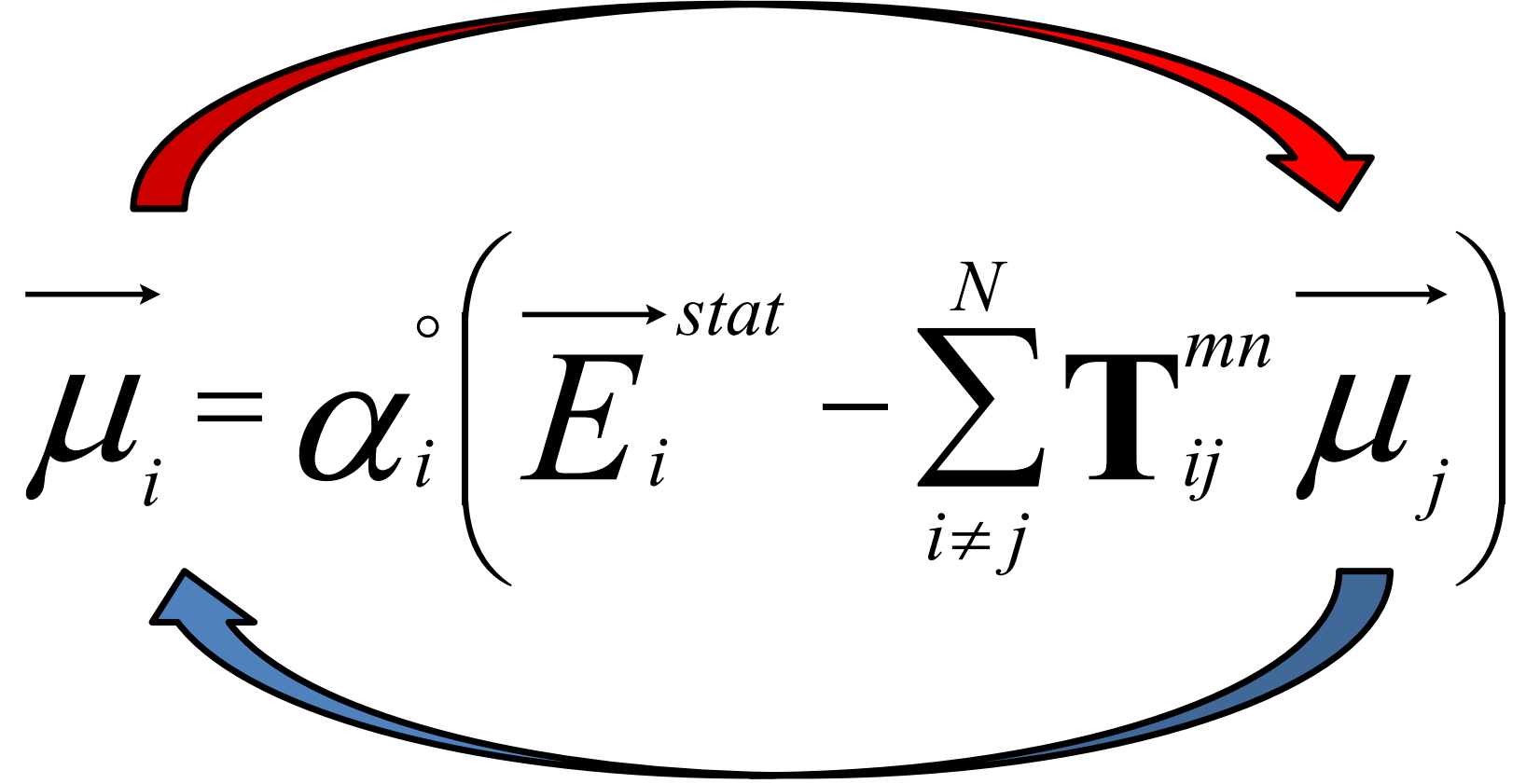

$$
U_{p o l}=-\frac{1}{2} \sum_{i}^{N} \overrightarrow{\boldsymbol{\mu}_{i}} \bullet{\overrightarrow{E_{i}}}^{\text {stat }}
$$




\section{$\mathrm{H}_{2}$ Models}

- Buch $^{1}$

- One-site model including only van der Waals interactions using a Lennard-Jones potential.

- BSS (BSS = Belof, Stern, Space) $)^{2}$

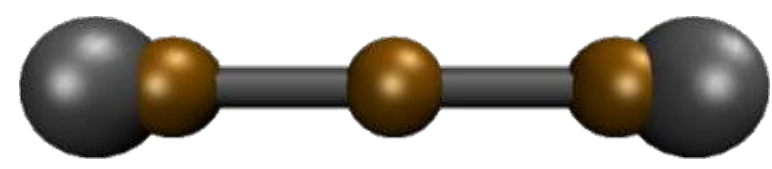

- Five-site model adding charge-quadrupole interactions.

- $\mathrm{DL}(\mathrm{DL}=\text { Darkrim and Levesque })^{3}$

- Three-site model with charge-quadrupole interactions.

- $\mathrm{BSSP}^{2}$

- Analogue of BSS model augmented with explicit manybody polarization. 


\section{Simulation Details}

- Grand Canonical Monte Carlo (GCMC)

- Periodic Boundary Conditions

- Many-Body Polarization via Thole-Applequist Model

- Massively Parallel Monte Carlo (MPMC) code (https://github.com/mpmccode/mpmc)

- Average particle number calculated by:

$$
\langle N\rangle=\frac{1}{\Xi} \sum_{N=0}^{\infty} e^{\beta \mu N}\left\{\prod_{i=1}^{3 N} \int_{-\infty}^{\infty} d x_{i}\right\} N e^{\beta \mu U\left(x_{1}, \cdots, x_{3 N}\right)}
$$

- Isosteric heat of adsorption $\left(Q_{\mathrm{st}}\right)$ calculated by:

$$
Q_{s t}=-\frac{\langle N U\rangle-\langle N\rangle\langle U\rangle}{\left\langle N^{2}\right\rangle-\langle N\rangle^{2}}+k T
$$




\section{Low Pressure Absolute $\mathrm{H}_{2}$ Adsorption Isotherms in Mg-MOF-74 at $77 \mathrm{~K}$}

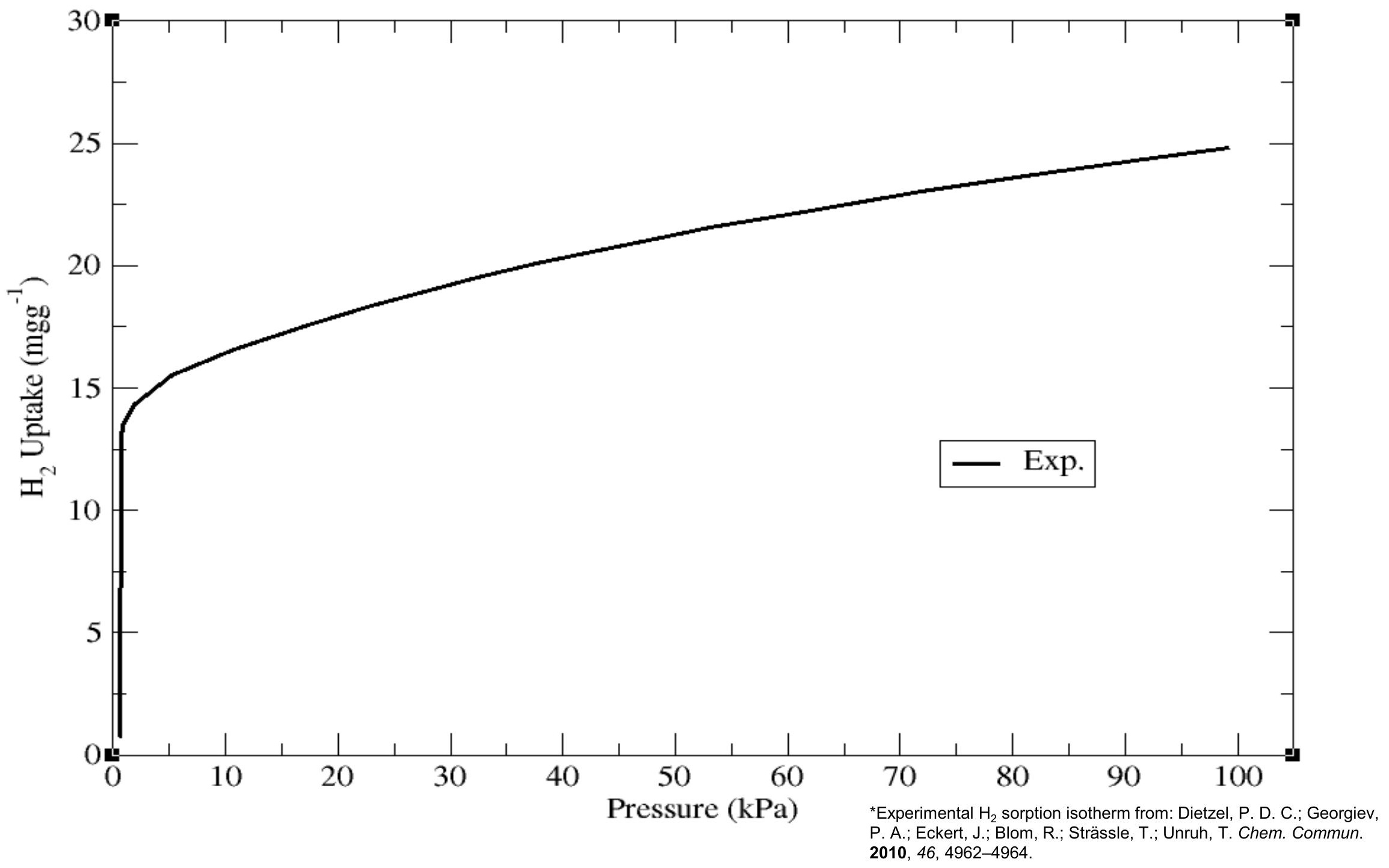




\section{Low Pressure Absolute $\mathrm{H}_{2}$ Adsorption Isotherms in Mg-MOF-74 at $77 \mathrm{~K}$}

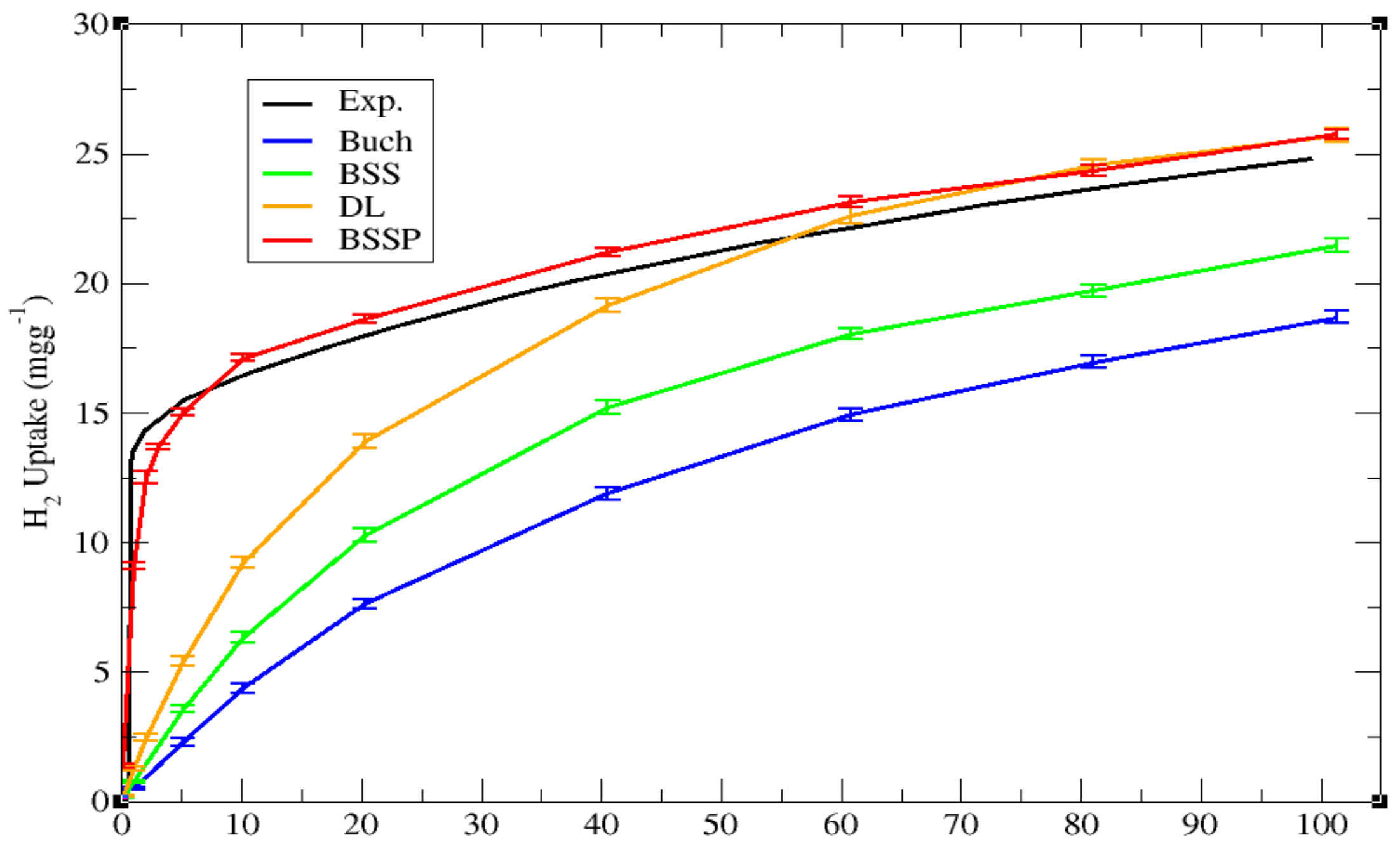

Pham, T.; et al. J. Phys. Chem. C 2014, 118, 22683-22690.

${ }^{*}$ Experimental $\mathrm{H}_{2}$ sorption isotherm from: Dietzel, P. D. C.; Georgiev, P. A.; Eckert, J.; Blom, R.; Strässle, T.; Unruh, T. Chem. Commun. 2010, 46, 4962-4964. 


\section{$Q_{\text {st }}$ For $\mathrm{H}_{2}$ in Mg-MOF-74}

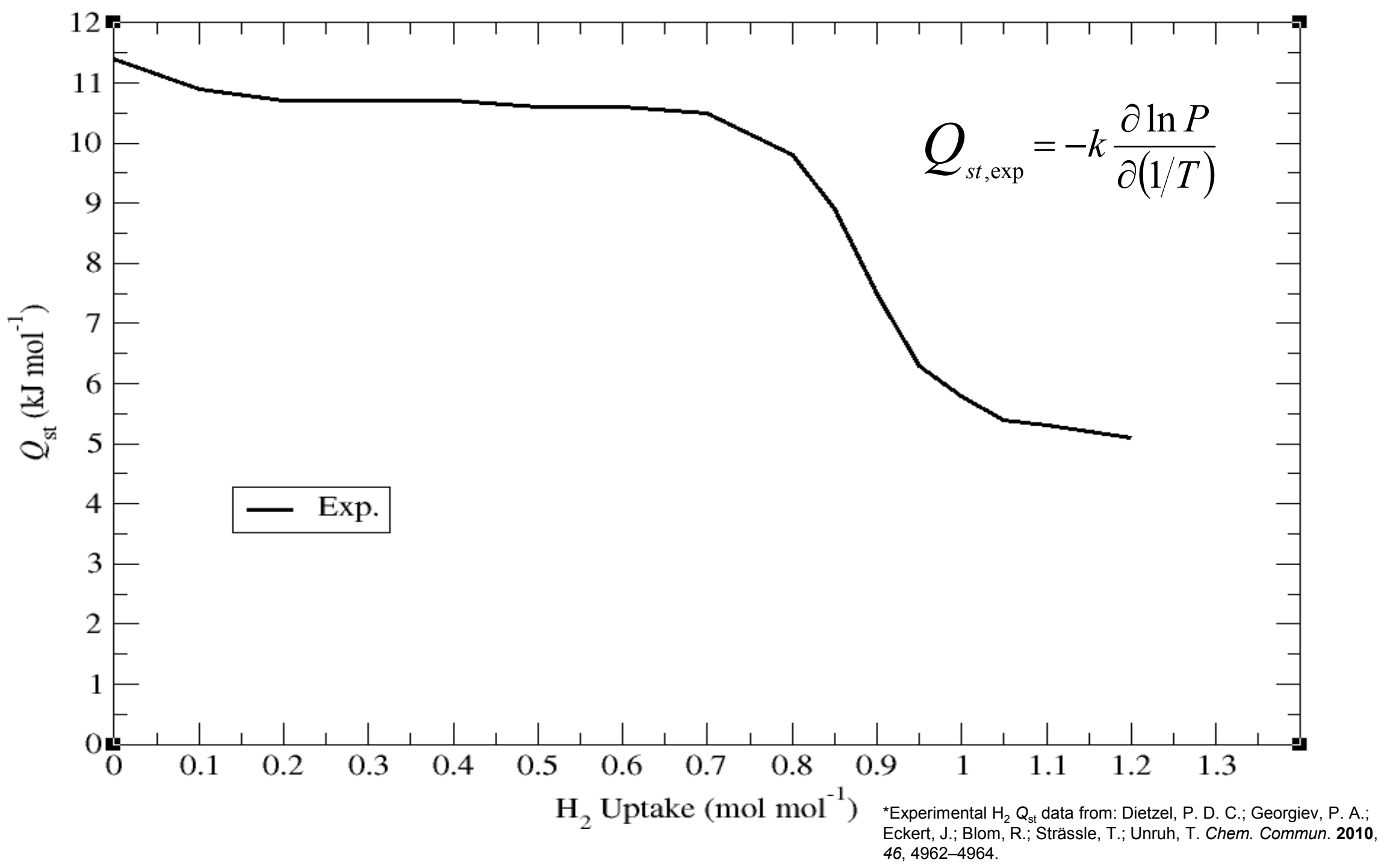




\section{$Q_{\text {st }}$ For $\mathrm{H}_{2}$ in Mg-MOF-74}

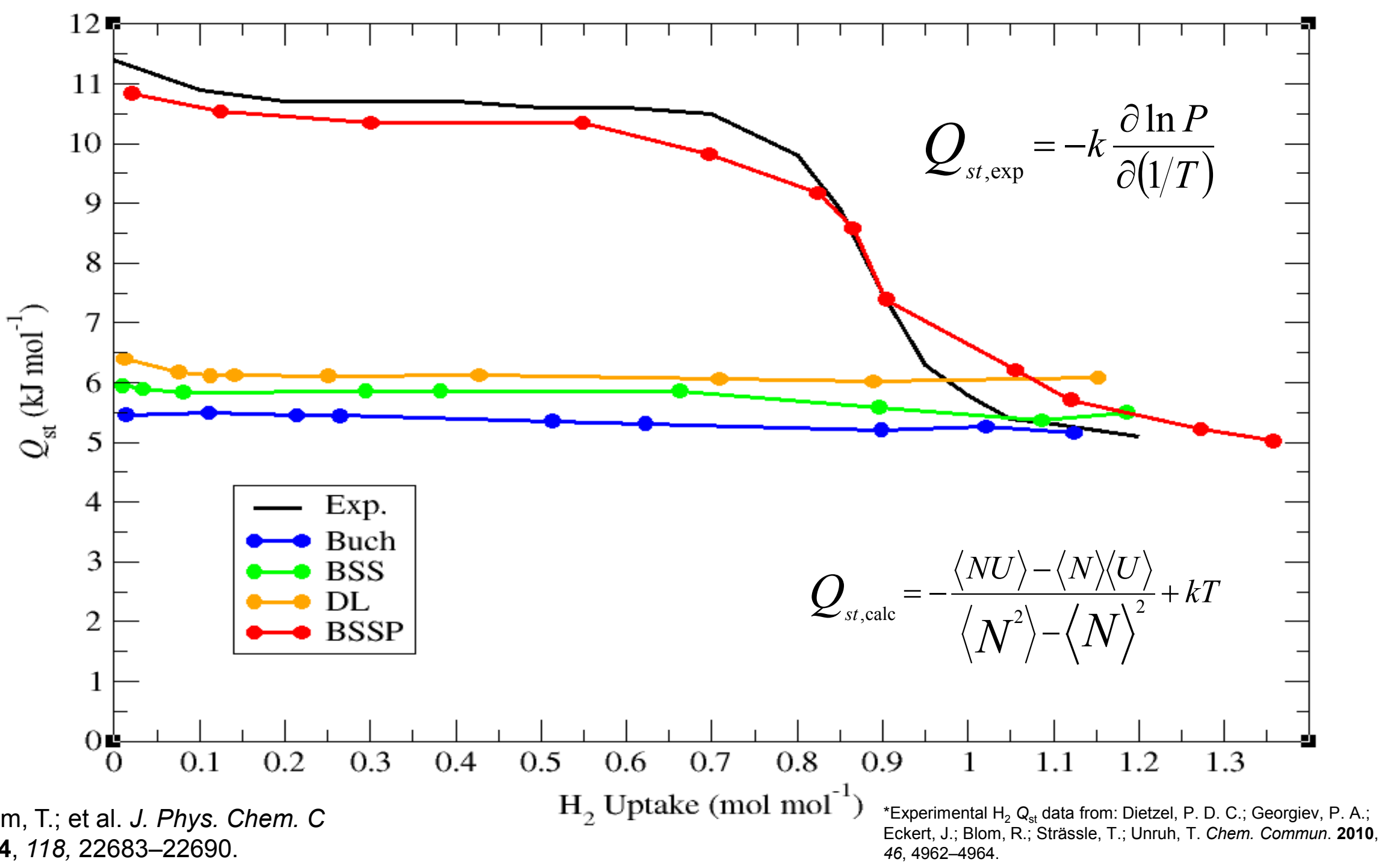

Pham, T.; et al. J. Phys. Chem. C 2014, 118, 22683-22690. 


\section{Radial Distribution Function Around $\mathrm{Mg}^{2+}$ lons in Mg-MOF-74 at $77 \mathrm{~K} / 0.01 \mathrm{~atm}$}

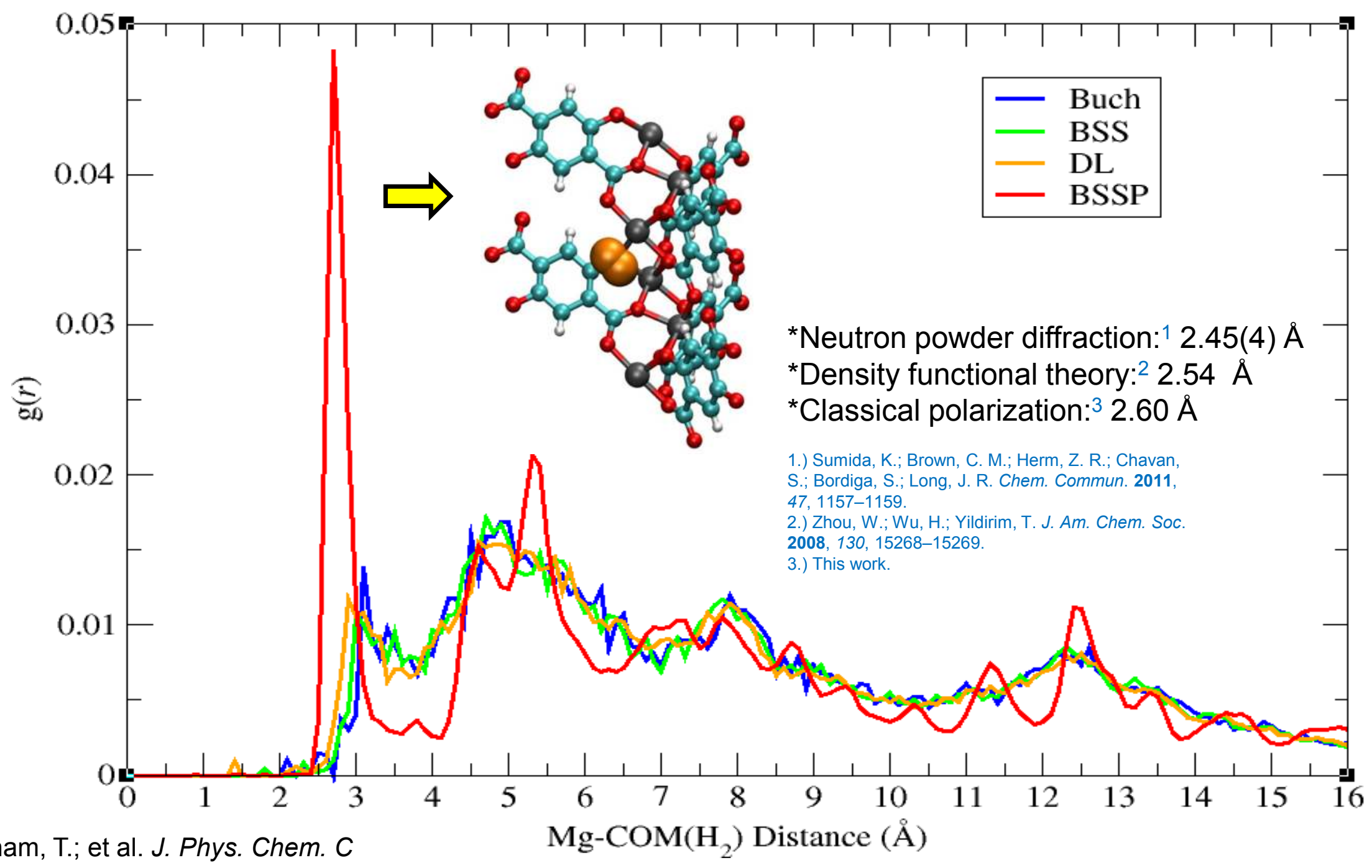

Pham, T.; et al. J. Phys. Chem. C 2014, 118, 22683-22690. 


\section{Low Pressure Absolute $\mathrm{H}_{2}$ Sorption Isotherms in M-MOF-74 Series at $77 \mathrm{~K}$}

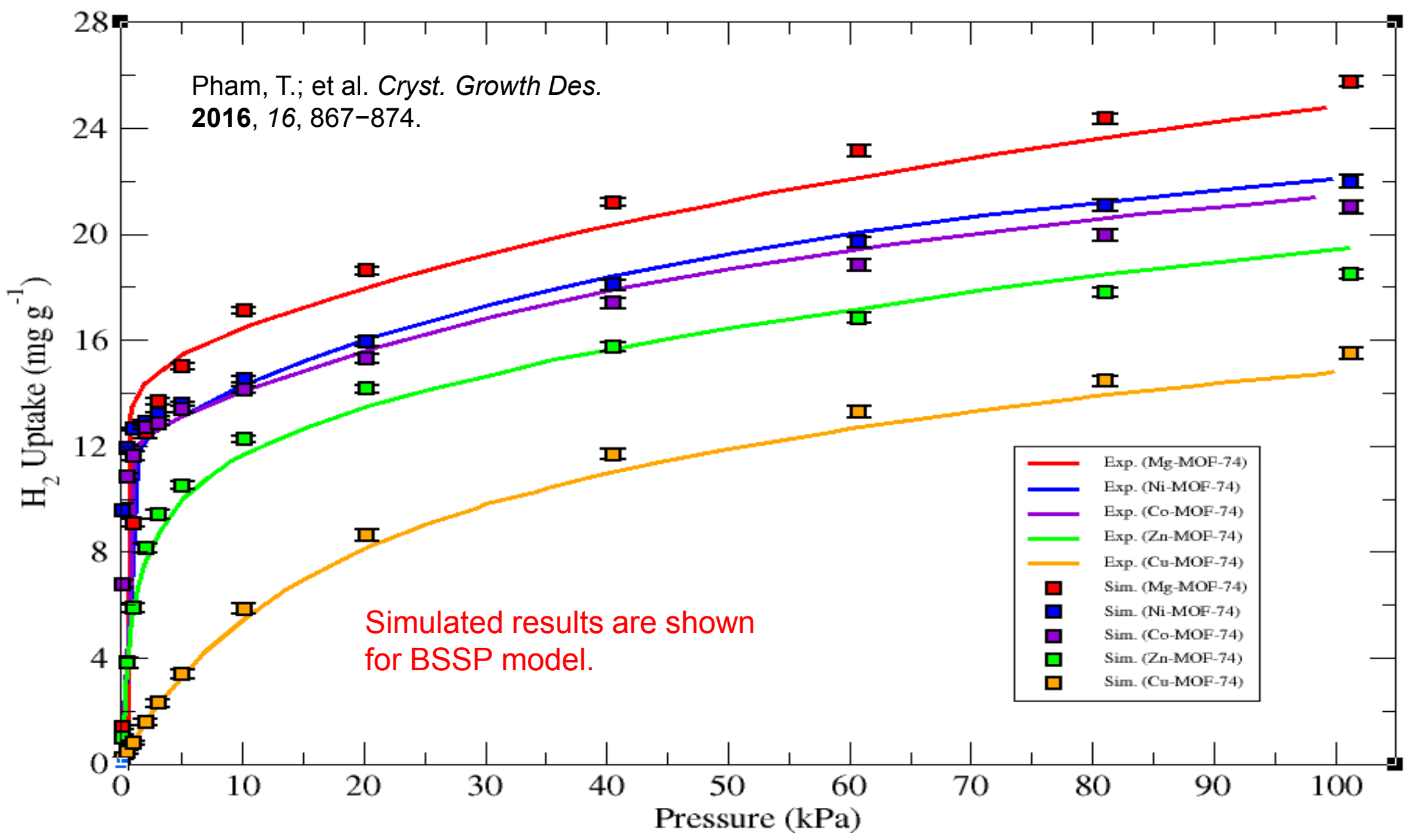

${ }^{*}$ Experimental $\mathrm{H}_{2}$ sorption isotherm for Mg-MOF-74, Ni-MOF-74, and Co-MOF-74 from: Dietzel, P. D. C.; et al. Chem. Commun. 2010, 46, 4962-4964. ${ }^{*}$ Experimental $\mathrm{H}_{2}$ sorption isotherm for Zn-MOF-74 from: Rowsell, J. L. C.; Yaghi, O. M. J. Am. Chem. Soc. 2006, 128, $1304-1315$.

${ }^{*}$ Experimental $\mathrm{H}_{2}$ sorption isotherm for Cu-MOF-74 from: Rosnes, M. H.; et al. J. Mater. Chem. A 2015, 3, 4827-4839. 


\section{$Q_{\text {st }}$ For $\mathrm{H}_{2}$ in M-MOF-74 Series}

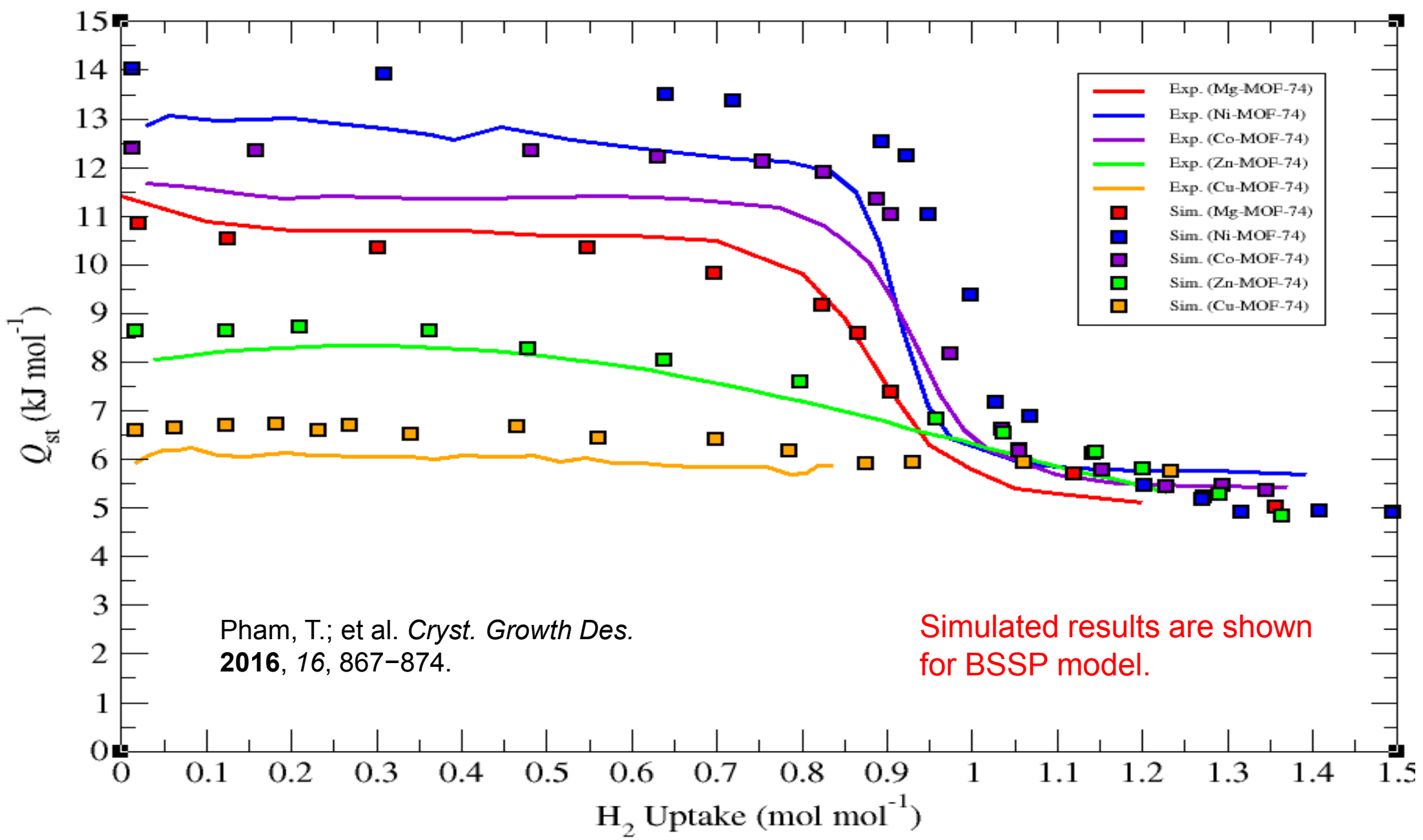

*Experimental $\mathrm{H}_{2}$ sorption isotherm for Mg-MOF-74, Ni-MOF-74, and Co-MOF-74 from: Dietzel, P. D. C.; et al. Chem. Commun. 2010, 46, $4962-4964$. *Experimental $\mathrm{H}_{2}$ sorption isotherm for Zn-MOF-74 from: Rowsell, J. L. C.; Yaghi, O. M. J. Am. Chem. Soc. 2006, 128, $1304-1315$.

*Experimental $\mathrm{H}_{2}$ sorption isotherm for Cu-MOF-74 from: Rosnes, M. H.; et al. J. Mater. Chem. A 2015, 3, 4827-4839. 


\section{Dipole Distribution in M-MOF-74 Series at $77 \mathrm{~K} / 0.20 \mathrm{~atm}$}

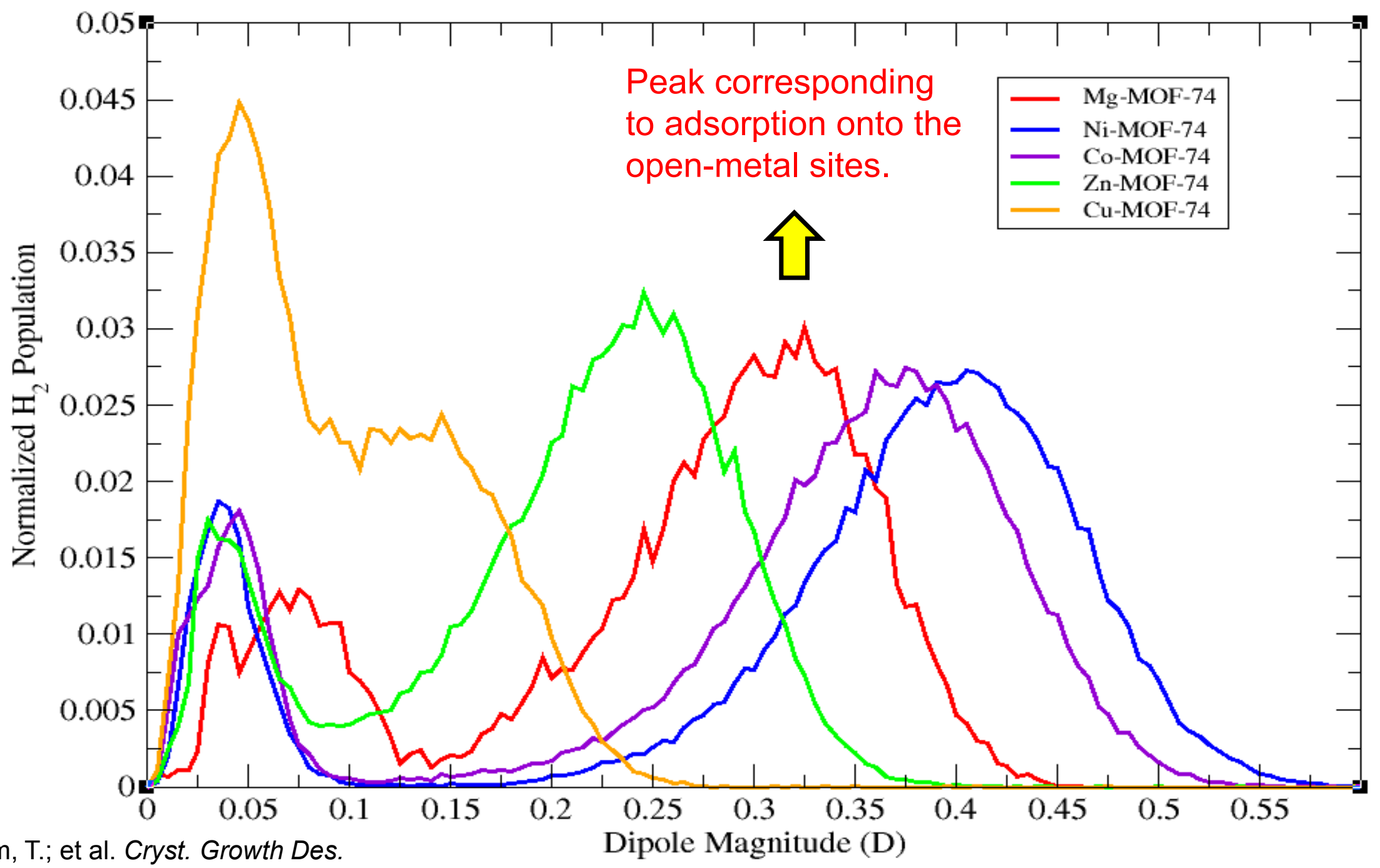


1.) Sumida, K.; et al.

Chem. Commun. 2011, 47, 1157-1159.

2.) Brown, C. M.; et al. Chem. Phys. 2013, 427, 3-8.

3.) Kapelewski, M. T. J. Am. Chem. Soc. 2014, 136, 12119-12129.

4.) Liu, Y.; et al. Langmuir 2008, 24, 4772-4777.

5.) Rosnes, M. H. J.

Mater. Chem. A 2015, 3, 4827-4839.

\section{$\mathrm{H}_{2}-$ Metal Distances in M-MOF-74 Series}

Strength of $\mathrm{M}^{2+}-\mathrm{H}_{2}$ Interaction:

$\left\{\begin{array}{l}\text { Ni-MOF-74 } \\ \text { Co-MOF-74 } \\ \text { Mg-MOF-74 } \\ \text { Zn-MOF-74 } \\ \text { Cu-MOF-74 }\end{array}\right.$

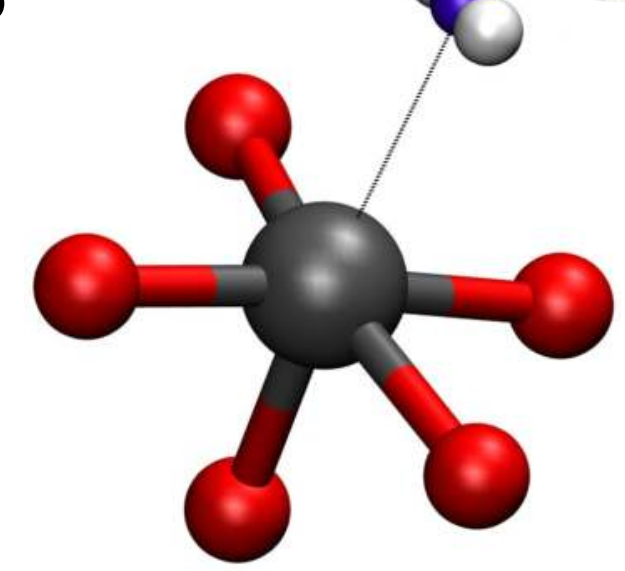

\begin{tabular}{|c|c|c|}
\hline MOF & \multicolumn{2}{|c|}{ M $^{2+}-\operatorname{COM}\left(\mathrm{H}_{2}\right)$} \\
\hline & Exp. $^{a}$ & Calc. $^{b}$ \\
\hline Mg-MOF-74 & $2.45(4)^{1}$ & 2.55007 \\
\hline Ni-MOF-74 & $2.20(1)^{2}$ & 2.34179 \\
\hline Co-MOF-74 & $2.32(2)^{3}$ & 2.45267 \\
\hline Zn-MOF-74 & $\sim 2.6^{4}$ & 2.67825 \\
\hline Cu-MOF-74 & $3.03(2)^{5}$ & 2.90199 \\
\hline
\end{tabular}

${ }^{a}$ Based on neutron powder diffraction ${ }^{b}$ Based on simulated annealing
Pham, T.; et al. J. Phys. Chem. C 2015, 119, 1078-1090.

Pham, T.; et al. Cryst. Growth Des. 2016, 16, 867-874. 


\section{Atomic Partial Charges and}

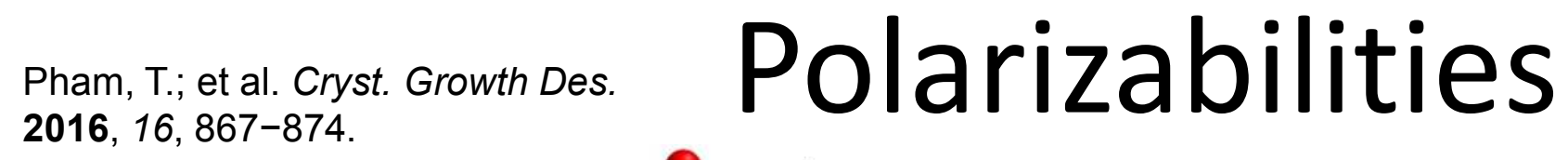

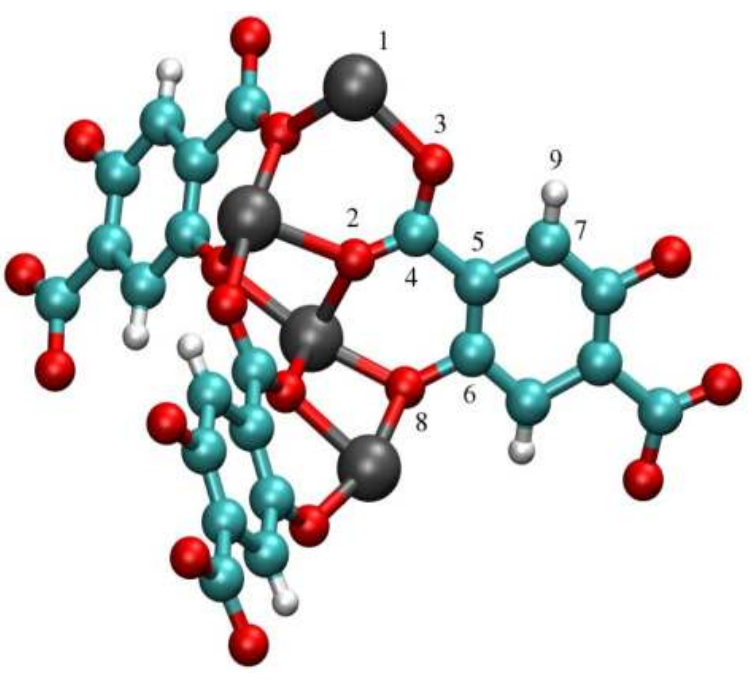

\begin{tabular}{|c|c|}
\hline $\mathrm{M}^{2+} \mathrm{Ion}$ & $\alpha^{\circ}\left(\AA^{3}\right)$ \\
\hline $\mathrm{Mg}^{2+}$ & 0.00720 \\
\hline $\mathrm{Ni}^{2+}$ & 2.94650 \\
\hline $\mathrm{Co}^{2+}$ & 3.26440 \\
\hline $\mathrm{Zn}^{2+}$ & 1.98870 \\
\hline $\mathrm{Cu}^{2+}$ & 2.19630 \\
\hline
\end{tabular}

\begin{tabular}{|c|c|c|c|c|c|c|}
\hline Atom & Label & $\mathrm{Mg}$ & $\mathrm{Ni}$ & $\mathrm{Co}$ & $\mathrm{Zn}$ & $\mathrm{Cu}$ \\
\hline $\mathbf{M}$ & $\mathbf{1}$ & 1.67800 & 1.50150 & 1.42010 & 1.49240 & 1.12860 \\
\hline $\mathbf{O}$ & $\mathbf{2}$ & -0.87140 & -0.78000 & -0.79450 & -0.82960 & -0.76640 \\
\hline $\mathbf{C}$ & $\mathbf{3}$ & -0.76800 & -0.66470 & -0.58940 & -0.75710 & -0.72140 \\
\hline $\mathbf{C}$ & $\mathbf{4}$ & 0.93030 & 0.88260 & 0.93940 & 0.87980 & 1.14250 \\
\hline $\mathbf{C}$ & $\mathbf{5}$ & -0.48150 & -0.44910 & -0.52700 & -0.26750 & -0.53740 \\
\hline $\mathbf{C}$ & $\mathbf{6}$ & 0.46750 & 0.44070 & 0.49000 & 0.40420 & 0.55200 \\
\hline $\mathbf{O}$ & $\mathbf{7}$ & -0.38240 & -0.43910 & -0.40320 & -0.37410 & -0.33180 \\
\hline $\mathbf{H}$ & $\mathbf{8}$ & -0.80540 & -0.71650 & -0.72910 & -0.77420 & -0.68620 \\
\hline
\end{tabular}




\section{Capturing the $\mathrm{H}_{2}-$ Metal Interaction in Mg-MOF-74 Using Classical} Polarization

Tony Pham, Katherine A. Forrest, Keith McLaughlin, Juergen Eckert, and Brian Space*

Department of Chemistry, University of South Florida, 4202 East Fowler Avenue, CHE205, Tampa, Florida 33620-5250, United States

THE JOURNAL OF

PHYSICAL CHEMISTRY

Understanding the $\mathrm{H}_{2}$ Sorption Trends in the M-MOF-74 Series ( $\mathrm{M}=$ $\mathrm{Mg}, \mathrm{Ni}, \mathrm{Co}, \mathrm{Zn}$ )

Tony Pham, ${ }^{\dagger}$ Katherine A. Forrest, ${ }^{\dagger}$ Rahul Banerjee, ${ }^{\dagger}$ Gisela Orcajo,, Juergen Eckert, ${ }^{\dagger}$ and Brian Space ${ }^{*}, \dot{\dagger}$

${ }^{\dagger}$ Department of Chemistry, University of South Florida, 4202 East Fowler Avenue, CHE205, Tampa, Florida 33620-5250, United States

${ }^{\ddagger}$ Physical/Materials Chemistry Division, CSIR-National Chemical Laboratory, Dr. Homi Bhabha Road, Pune 411008, India

"Department of Chemical and Energy Technology, ESCET, Rey Juan Carlos University, C/Tulipán s/n, 28933 Móstoles, Madrid, Spain

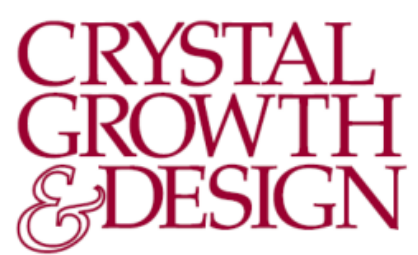

Dramatic Effect of the Electrostatic Parameters on $\mathrm{H}_{2}$ Sorption in an M-MOF-74 Analogue

Tony Pham, Katherine A. Forrest, Juergen Eckert, and Brian Space* 


\section{Acknowledgments}

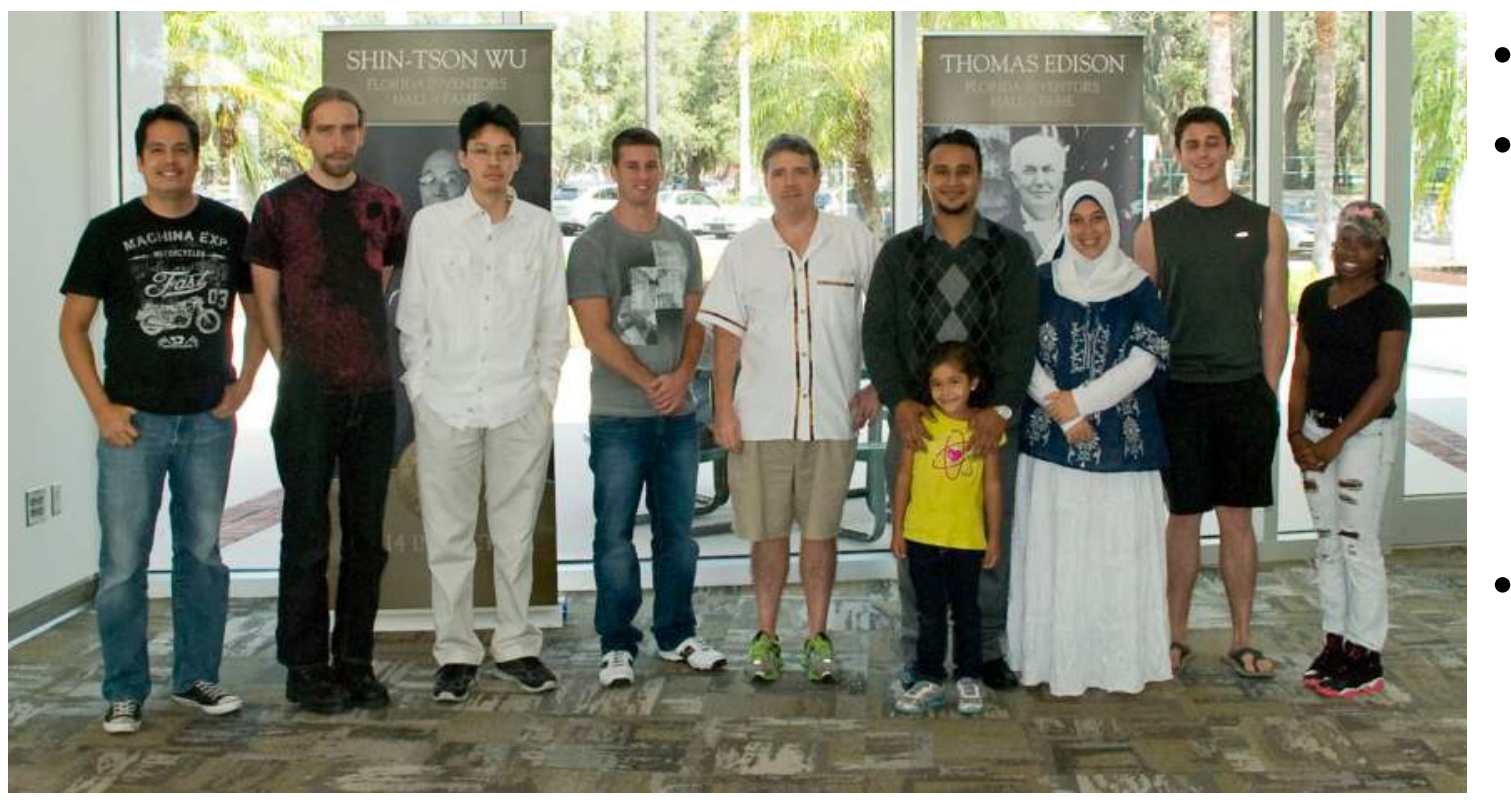

- Professor: Brian Space

- Group Members (2014-2016):

- Katherine A. Forrest

- Christian R. Cioce

- Brant Tudor

- Adam Hogan

- Douglas M. Franz

- Collaborator: Juergen Eckert

TJST $\frac{\text { WNWERSITY OH }}{\text { SOUHH FLORIDA }}$

Researcla Computing

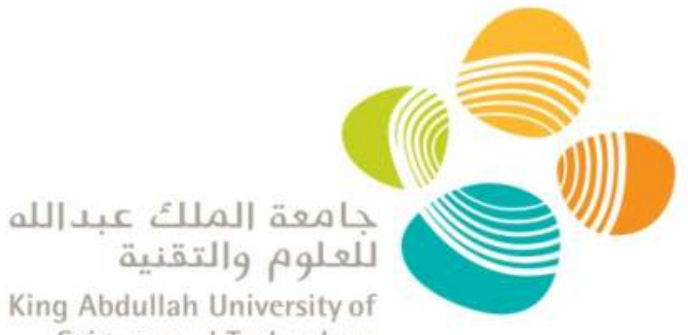

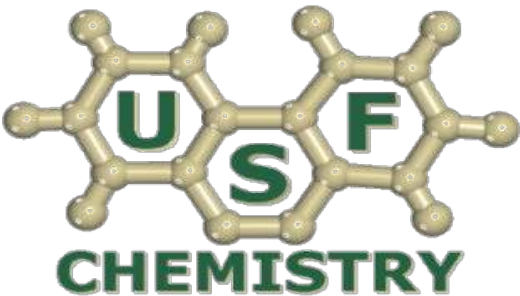

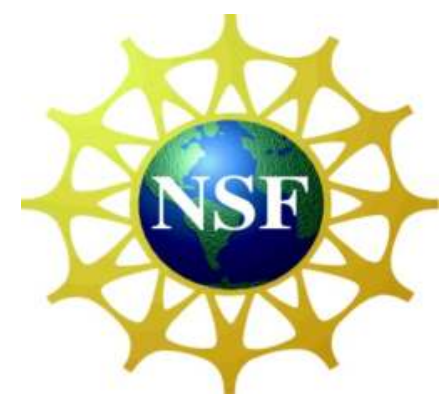

다ㅇㅛㅛ

G TeraGrid

XSEDE

Extreme Science and Engineering Discovery Environment 